\title{
Engaging community stakeholders in research on best practices for clinical genomic sequencing
}

\author{
Ida Griesemer ${ }^{* 1,2}$ (D), Brooke S Staley ${ }^{(\mathbb{D})}$, Alexandra F Lightfoot ${ }^{1,4}(\mathbb{D})$, Lizzy Bain ${ }^{5}$, Derrick \\ Byrd $^{6}$, Carol Conway ${ }^{7}$, Tracey L Grant ${ }^{8}$, Barbara Leach ${ }^{9}$, Laura V Milko ${ }^{8}$ iD, Lonna \\ Mollison $^{8}$, Nadiah Porter ${ }^{10}$, Sharron Reid ${ }^{11}$, Gerri Smith ${ }^{12}$, Margaret Waltz ${ }^{13}$, Jonathan S \\ Berg $^{8}$ (D), Christine Rini ${ }^{14}$ \& Julianne M O’Daniel ${ }^{8}$ (iD) \\ ${ }^{1}$ Department of Health Behavior, UNC Chapel Hill, Chapel Hill, NC, USA \\ ${ }^{2}$ Cecil G. Sheps Center for Health Services Research, UNC Chapel Hill, Chapel Hill NC, USA \\ ${ }^{3}$ Department of Epidemiology, UNC Chapel Hill, Chapel Hill, NC, USA \\ ${ }^{4}$ Center for Health Promotion \& Disease Prevention, UNC Chapel Hill, Chapel Hill, NC, USA \\ ${ }^{5}$ Parent/Advocate, Knightdale, NC, USA \\ ${ }^{6}$ Parent/Advocate, Family Resource Center South Atlantic, Raleigh, NC, USA \\ ${ }^{7}$ Parent/Advocate, Parent Advocates for Adult Children with Intellectual \&/or Developmental Disabilities in NC, Chapel Hill, NC, \\ USA \\ ${ }^{8}$ Department of Genetics, UNC Chapel Hill, Chapel Hill, NC, USA \\ ${ }^{9}$ Parent/Advocate, Family Support Program, School of Social Work, UNC Chapel Hill, Chapel Hill, NC, USA \\ ${ }^{10}$ Parent/Advocate, Durham, NC, USA \\ ${ }^{11}$ Parent/Advocate, Wake County Sickle Cell Support Group, Raleigh, NC, USA \\ ${ }^{12}$ Parent/Advocate, Holly Springs, NC, USA \\ ${ }^{13}$ Department of Social Medicine, UNC Chapel Hill, Chapel Hill, NC, USA \\ ${ }^{14}$ Department of Medical Social Sciences, Feinberg School of Medicine \& Robert H. Lurie Comprehensive Cancer Center of \\ Northwestern University, Chicago, IL, USA \\ *Author for correspondence: Tel.: +603 667 1473; idajane@email.unc.edu
}

\begin{abstract}
Aim: Maximizing the utility and equity of genomic sequencing integration in clinical care requires engaging patients, their families, and communities. The NCGENES 2 study explores the impact of engagement between clinicians and caregivers of children with undiagnosed conditions in the context of a diagnostic genomic sequencing study. Methods: A Community Consult Team (CCT) of diverse parents and advocates for children with genetic and/or neurodevelopmental conditions was formed. Results: Early and consistent engagement with the CCT resulted in adaptations to study protocol and materials relevant to this unique study population. Discussion: This study demonstrates valuable contributions of community stakeholders to inform the implementation of translational genomics research for diverse participants.
\end{abstract}

First draft submitted: 22 May 2020; Accepted for publication: 28 July 2020; Published online:

7 October 2020

Keywords: clinical trial $\bullet$ community-academic partnerships $\bullet$ community engagement $\bullet$ diagnostic odyssey $\bullet$ genetics $\bullet$ genomic sequencing $\bullet$ health equity $\bullet$ health services research $\bullet$ precision medicine $\bullet$ underserved populations

As evidence for its clinical usefulness grows [1,2] and costs decrease [3], genomic sequencing is rapidly being incorporated into clinical care. Research on best practices for the clinical application of genomics is essential to the effective translation of precision medicine in clinic settings [4], and to guide clinical genomics policy [5]. However, traditional research methods have led to the overrepresentation of people with European ancestry and with higher socioeconomic status in clinical genomics studies [6,7]. To improve diversity in genomics research and ensure population benefits across racial, ethnic, and socioeconomic subgroups, targeted approaches to enhance inclusion of diverse study cohorts are necessary. As a result, there is growing recognition of the need to engage stakeholders from historically underserved and underrepresented groups early in the design and implementation of genomics research $[8,9]$. Early engagement of community stakeholders in research processes can result in the identification of potential cultural and practical barriers that may otherwise be unidentified by research teams. Additionally, 
community stakeholders can help research teams develop methods for eliminating barriers to recruiting and retaining diverse study cohorts.

Building research partnerships with community stakeholders can be time-intensive, requiring effort from both researchers and community members to build trust and navigate power dynamics [10,11]. Researchers can facilitate this process by recognizing the importance of collaborating with families and individuals who may be directly impacted by genomic research. To establish these relationships, researchers must indicate their desire and willingness to engage underserved and underrepresented stakeholders in inclusive relationships that value their experiences and perspectives. Researchers must also recognize and acknowledge historical medical research that exploited individuals from communities of color without disclosing their true research agendas and with reckless disregard for participants' well-being [12,13]. Among the African American community, for instance, the legacy of the US Public Health Service Syphilis Study at Tuskegee, in which researchers withheld syphilis treatment from African American participants, remains a driver of mistrust of medical research [14,15]. The history of the eugenics movement, which sought to legitimize racist strategies to classify and oppress human beings through methods such as forced sterilization, is tied directly to the origins of modern human genetics and genomics research [16]. Additionally, the ethical violations surrounding the story of Henrietta Lacks, an African American woman whose cells were taken without her permission and have been widely used in biomedical research, have been documented in a popular book and are now widely known to the public [17]. This history and legacy of mistrust continues to influence the reluctance of community members to participate in clinical genomics research, and researchers often fail to dedicate sufficient time and resources to reach out to underrepresented communities to give them an equitable opportunity to participate [18-20]. Members of these communities may be receptive to research participation if they are provided with transparent information about the study and are informed of any benefits.

Beyond these historical barriers, underserved and underrepresented patients and caregivers also face logistical challenges making it difficult to participate in clinical genomic research. First, the location of clinics present barriers for families with limited access to private or public transportation. Second, families engaging in clinical genomics must coordinate care across a complex, fragmented system, and navigate appointments with multiple providers and specialties. This navigation becomes increasingly challenging when additional research demands, such as surveys or blood draws, are added to healthcare visits. Participating in research often requires spending extra time at the clinic, meaning patients and caregivers might take more time off from school or work. Clinic materials are also often written at a health literacy level that can make the information inaccessible to patients and caregivers, an issue particularly relevant in genetic medicine [21]. The use of medical jargon can make it difficult for families to understand research goals and the value of their participation.

While there are substantial challenges to increasing diversity in clinical genomics research, engaging community stakeholders is a critical strategy for mitigating those challenges to improve research efforts. Yet there are few examples of studies that engage community stakeholders in the clinical genomics research process [9]. Clinical genomics community stakeholders might include people with undiagnosed conditions seeking health information, people with disabilities of unknown etiology, caregivers or parents of people with genetic conditions, and other community health advocates. Because of their first-hand knowledge and experiences, community members can offer essential advice and help guide research decisions related to recruitment strategy, education materials, study logistics, data collection instruments, and participant incentives. Their insight can also contribute to analyses and guide broad dissemination strategies. Therefore, stakeholder engagement has the potential to strengthen intervention designs and increase representation of underserved and underrepresented communities in genomics research.

This paper describes the stakeholder engagement process and outcomes from the NCGENES 2 study, a randomized control trial evaluating the clinical utility of genomic sequencing. The original NCGENES study explored the challenges and feasibility of integrating genomic medicine into clinical care [22]. The current study builds on the knowledge gained from the original by exploring the engagement processes between clinicians and caregivers of children with undiagnosed conditions and the potential benefit of exome sequencing over usual clinical care for pediatric patients with unknown yet likely genetic disorders. The research team took several measures to form a community-academic team that could inform processes for meaningful engagement of NCGENES 2 participants. These strategies, described below, allowed for robust involvement of community stakeholders to provide consultation and advice to inform study processes. 


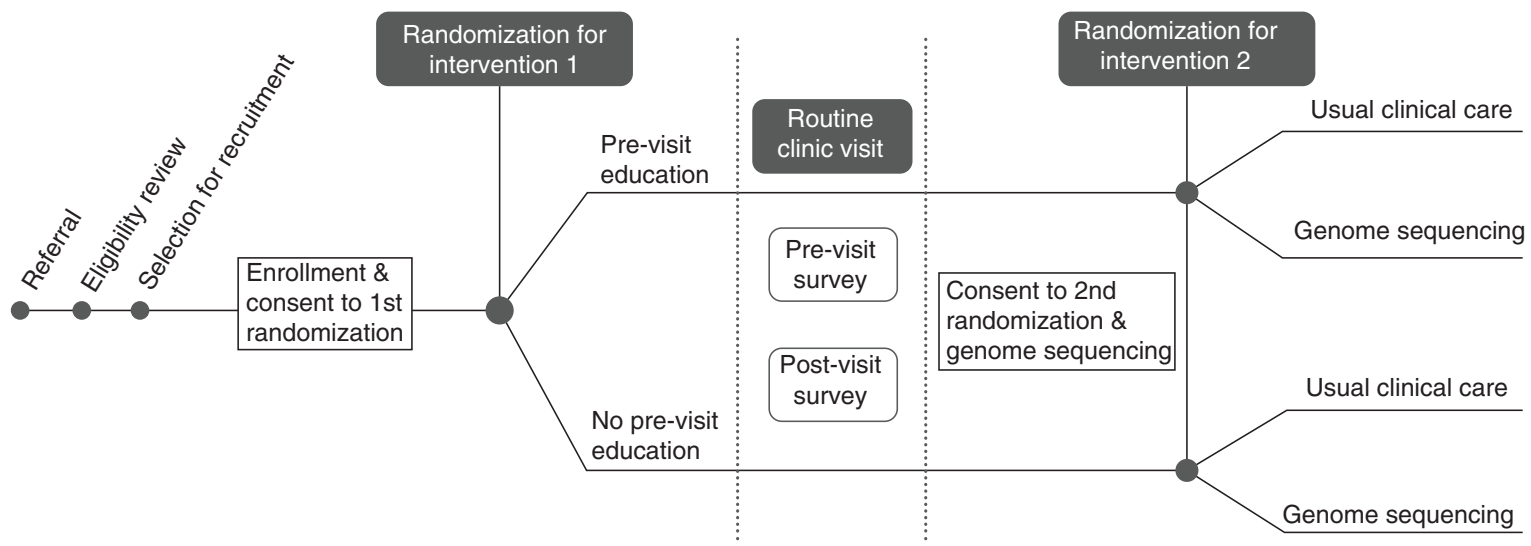

Figure 1. NCGENES 2 study design. All eligible participants are new patients presenting for evaluation to pediatric genetics or pediatric neurology clinics. Enrollment is completed by phone prior to the scheduled new patient visit. The first randomization is +/- Pre-Visit Education materials which are mailed. Following the new patient visit, parents/participants consent/assent to the second randomization: +/- Genome Sequencing in addition to any tests their doctor ordered. Additional surveys occur after second randomization which are not depicted here. Further, the Pre-Visit Education arm receives a second mailed education packet focused on understanding test results.

\section{Methods}

To enhance diversity in genomics research, the NCGENES 2 study aims to enroll at least 60\% of study participants from medically underserved (i.e., eligible for Medicaid or North Carolina Children's Health Insurance Program) or historically underrepresented minority populations (i.e., Hispanic or non-White/European American). The study is testing patient advocacy and engagement strategies, such as caregiver education and question prompt lists, to build evidence for best practices to improve clinician-caregiver communication around genomic sequencing. Figure 1 presents an overview of the study design. All study procedures were approved by the University of North Carolina at Chapel Hill's Institutional Review Board (IRB), and participants provided informed consent to take part in NCGENES 2.

To incorporate community stakeholder perspectives into study design and process decisions, we formed a Community Consult Team (CCT) of members who agreed to participate in the research team. The IRB specified an exempt status for the CCT engagement process.

\section{Recruitment}

Our study sought to engage 6-10 members for the CCT. We aimed to recruit parents and advocates for children with genetic and/or neurodevelopmental conditions. Additionally, given the research study's goals for engaging diverse populations, special emphasis was placed on recruiting members from historically underserved or underrepresented communities. Members were primarily recruited through two contacts: attendance at an earlier pilot meeting to review a study enrollment phone script and contact through a central family advocate. The pilot meeting included members of a local Sickle Cell support group and individuals known to one of the study researchers (AL). The central family advocate was identified through an internet search of local resources for families with children who have special needs. Based on their advocacy role, this individual was well positioned to be a key recruiter and contributing member of the CCT. All but one CCT member were recruited over a two-month period during the early stages of study development.

\section{Results}

\section{Participants}

The NCGENES 2 CCT includes seven members who collectively bring a broad range of professional and lived experience to the study. The CCT members describe themselves via the following categories: six are parents of children who have had medical needs (child age range 6-44); five have had genetic evaluation for their child(ren); five identify as Black/African American and two as White/Caucasian; six are female and one is male. The CCT members live and work in central North Carolina and have valuable professional and volunteer perspective including: serving on committees and as board members for disability advocacy organizations, coordinating child protective services 


\begin{tabular}{|l|l|}
\hline $\begin{array}{l}\text { Table 1. Community engagement strategies and tangible outcomes from the NCGENES } 2 \text { Study. } \\
\text { Strategy/study area }\end{array}$ & Example \\
\hline Engagement strategies & \\
\hline 1. Relationship building & Creating a 'group resume' in the initial meeting to highlight CCT members' strengths and expertise \\
\hline 2. Flexible protocols & Allowing for changes to the study protocol to meaningfully incorporate feedback from the CCT \\
\hline 3. Flexible meetings & Rotating the time and location of CCT meetings \\
\hline 4. Compensation & Paying CCT members for their time and effort \\
\hline 5. Inclusive dissemination & Partnering with CCT members to frame messages around research findings for dissemination \\
\hline Revised study areas based on CCT feedback & \\
\hline 1. Recruitment language & Incorporating CCT feedback to use inclusive language in recruitment scripts \\
\hline 2. Education materials & Reframing messages in educational booklets to simplify themes and enhance trust around genetic testing \\
\hline 3. Survey instruments & Removing burdensome survey measures \\
\hline 4. Compensation and gifts & $\begin{array}{l}\text { Offering child participants gifts such as a t-shirt or backpack as a token of appreciation for their participation in the } \\
\text { research study }\end{array}$ \\
\hline
\end{tabular}

CCT: Community Consult Team

for the county, working as a family support specialist and mental health crisis counselor, volunteering with the Special Olympics, and serving on state-level committees to inform policy development for families and children who have disabilities.

\section{Engagement strategies}

The engagement strategies and tangible outcomes described below are summarized in Table 1. Relationship building

Establishing a small core group of community partners and a subgroup of study personnel who consistently attended CCT meetings encouraged familiarity among group members. We grounded the initial meeting in a strengthsbased approach by creating a 'group resume' to highlight group members' experiences, values, and motivations for being involved with the research study, which has been described as a strategy for building community-academic research partnerships [23]. An early focus on the strengths of community members helped balance power dynamics by acknowledging community members' expertise in their roles as caregivers and advocates.

Early meetings were longer and more frequent (approximately two hours, once a month, versus one hour per quarter as the study progressed) to nurture a foundation of trust. Central concepts considered essential to study development were defined and discussed. These included terms such as: the IRB, compensation, gene, genomic, secondary finding, and variant. This capacity-building exercise encouraged comfort with study personnel and CCT members as we learned together.

\section{Flexible protocols}

Early in study implementation, we used a flexible study protocol and timeline to incorporate changes based on input from the CCT. Partnering with the CCT early in the study enabled members, drawing on their lived and/or professional experience, to contribute to study protocol decisions and guide study material development. The incorporation of CCT feedback in study elements was highlighted in later meetings with members, demonstrating the value the research team placed on the CCT's perspective.

\section{Flexible meetings}

We rotated the location and time of the CCT meetings, often holding them in settings suggested by stakeholders, so the same group members were not required to travel long distances for every meeting. We also offered CCT members the option to participate remotely via phone. If CCT members had difficulty arranging childcare their children were welcome at the meetings. In advance of each meeting we sent CCT members study materials that we planned to review in the meeting. This led to more efficient use of meeting time as CCT members reviewed materials and prepared feedback in advance. 


\section{Compensation}

We compensated CCT members for meeting attendance, and we sought members' opinions regarding the format for monetary compensation (e.g., gift card, check, or cash). We also provided a meal at every in-person meeting since we often met in the evening. Food preferences were sought and integrated into menu planning.

\section{Inclusive dissemination}

The study took an inclusive approach to dissemination by partnering with CCT members to frame messages around the research findings and implications. We collaborated with CCT members on the dissemination of results from the stakeholder engagement process by developing various presentations and this manuscript together. CCT and research team members have co-presented study results at several local conferences and at the 2019 American Public Health Association's Annual Meeting in Philadelphia, PA, USA [24].

\section{Tangible outcomes}

These strategies have led to productive stakeholder engagement and vital feedback provided by the CCT, which resulted in the following study-related changes.

\section{Recruitment language}

Given the legacy of mistrust around medical research, CCT members emphasized the importance of clearly stating the study's goals during recruitment phone calls to curtail any skepticism participants may have about researchers' intentions. The CCT provided guidance on specific wording to use in recruitment scripts, simplifying the language to enhance the sense of transparency around the study's objective and intended outcomes. For example, the CCT suggested introducing the study goals by saying, "Ok, now let me tell you what the study is trying to do," and concluding recruitment by summing up the study in plain language. When it appeared that a disproportionate percentage of underserved/underrepresented caregivers were not returning the enrollment call, the CCT suggested the voicemail script be changed to mention that we were trying to reach the child's caregiver. This change was based on the CCT's observation that caregivers might be more likely to return a call if they knew it was related to their child.

\section{Education materials}

One of the intervention strategies tested in NCGENES 2 includes an educational booklet mailed to caregivers prior to their child's appointment. The CCT offered feedback on the language in the booklet, which prompted major revisions improving the booklet's readability and enhancing the idea that the appointment should be a partnership between clinicians and caregivers. In one section of the booklet titled, "Why was my child referred to this clinic?" the original text read, "Your child was referred because doctors think that he or she might have a genetic condition." The CCT felt that the phrase 'genetic condition' might prompt some underserved caregivers to become suspicious and distrustful of the reason for genetic testing given the historical context of eugenics. The CCT also thought that caregivers, seeking to understand more about genetic testing, would likely search the internet for answers where they might find confusing, overwhelming, or unreliable information. The group suggested revising this section to explain the reason for testing, which would promote caregivers' understanding and trust, and improve family engagement. After re-working the section together, the revised text read, "Your child was referred because there is a concern that he or she may have a health problem or be developing differently than other children. Sometimes parents have a concern that they ask their child's doctor about, leading to a referral to a pediatric specialist doctor. Other times a doctor has a concern during a regular check-up and recommends a visit to a pediatric specialist doctor." Beyond the booklet's text, the CCT provided feedback on the layout, including the need to ensure images be culturally relevant and not stereotypical. The format of the document was further revised to provide bullets and headings for improved readability.

The NCGENES 2 study is also evaluating the usefulness of a question prompt list as a strategy for enhancing clinician-caregiver engagement during the clinic appointment and when discussing results from any testing. Prior to their clinic visit, caregivers are mailed a list of questions they might have for their provider along with the booklet. Similarly, a booklet and list of questions focused on understanding test results is mailed after the appointment. Caregivers are encouraged to mark questions from the list they want to ask, bring the list to the clinic, and refer to it during their interactions with clinicians so they can gain information they want or need. The CCT used plain language to revise the questions on the prompt list and narrowed the questions down to main concerns that may be 
common across caregivers. Feedback from CCT members suggested it was important to provide two copies of the question prompt list for the clinic visit, so the caregiver could give one copy to the provider and keep the other for their own reference. The study team implemented this change to enhance the likelihood that the question prompt list would lead to enhanced communication between providers and caregivers.

\section{Survey instruments}

The research team developed surveys for caregivers participating in the study. CCT members reviewed and piloted these surveys to see how long they took to complete. CCT members also gave feedback on items that seemed repetitive. For example, the research team developed a self-efficacy measure with questions about how confident participants are in their ability to explain concepts such as diagnostic testing, their child's condition, or their child's test results. CCT members thought this measure was burdensome and repetitive as some concepts were so similar (e.g., diagnostic testing in general vs diagnostic testing their child received) that participants might not see them as distinct enough to yield meaningful results. The research team integrated this feedback while revising the measure of 30 items down to 11 .

Some surveys were originally going to be delivered over the phone. Because of the length of the surveys, CCT members said it would be very difficult for caregivers to dedicate $45 \mathrm{~min}$ to a phone call without interruptions. They suggested giving participants the survey either online or on paper so that participants could complete them at times that were convenient. The study team implemented this change to paper surveys. CCT members then provided feedback on survey formatting so that participants did not skip questions or feel too overwhelmed by the survey itself.

\section{Compensation \& gifts}

Other changes implemented in the study as a result of the CCT revolved around compensation. Gift cards have become a default mechanism for compensating study participants for many reasons including ease of tracking and security. However, the CCT provided feedback about potential drawbacks to gift cards (e.g., store location and shopping preference) and benefits of providing cash (e.g., ease for immediate use). Additionally, a member expressed that we were offering adequate incentives for caregiver participants, but the study did not provide any compensation to the children beyond offering snacks and videos during the study's clinic visit. It was noted that some children might not be able to eat the snack due to dietary restrictions or fasting requirements for their clinic visit and that some children may not have interest or ability to watch the videos. With this feedback, the study team decided to offer a variety of gifts to children at the end of their clinical research visit, such as a t-shirt, football or backpack. Study participants do not know about this aspect of the study until the end of their clinical visit, and the gift has been a meaningful surprise for caregivers who have participated in the research study thus far. Caregivers have mentioned that they appreciate that the research team considered the children and that a variety of gifts are available with appropriate options for various ages and developmental and physical capabilities.

\section{CCT member experiences}

The CCT process has also yielded benefits to the CCT members themselves. When asked about what they have gotten out of the process, CCT members expressed appreciation for the opportunity to share their views on genomics research, to be heard by researchers and to see their input affect change in research implementation. One member expressed that contributing to the study process felt like a way of 'pulling back the power' in that CCT members provided input that influenced the research approach. One CCT member expressed her appreciation for the flexible nature of the meetings that allowed her to bring her children when she had an issue with childcare. She said, "This is significant for me because although it was not ideal to have children present during our meetings, the sense of community that existed between CCT members and the research team was such that I did not feel judged and trusted that my children would not just be tolerated, but welcome." The group also appreciated that CCT meetings allowed them to connect with a community of caregivers with shared experiences. One CCT member said, "I like hearing other perspectives, it expands my thinking. I like to be informed. I can take it and share with someone and offer support to someone who might need that support." For this person, connecting with other CCT members made her feel more prepared to support other members of her community.

The CCT also noted that ongoing involvement in the research process, and later in dissemination efforts, was an important aspect of the experience. They emphasized that a large part of what made participating in the CCT a positive experience was that it was not just a 'one and done' where they were invited to give input once and then 
never contacted again. Some members compared the CCT to past experiences consulting for research projects, where they were invited toward the end of the project after major decisions were finalized to 'sign off' on the project. They contrasted those past experiences with NCGENES 2, noting that they were brought on in the early stages of the study, were consulted throughout the process, and were invited to participate in dissemination efforts. The ongoing involvement was positive because it allowed them to form a community with one another, and to see their recommendations reflected in tangible changes to the study process and materials.

\section{Discussion}

The NCGENES 2 study prioritized engagement of underserved and underrepresented stakeholders in genomics research by forming a Community Consult Team (CCT). The CCT was recruited during the developmental phase for the study, continues to meet regularly throughout the research process, and has been involved in dissemination efforts. CCT members offered recommendations on changes to intervention implementation, education materials, survey instruments, and dissemination products, which researchers incorporated into the study. By meeting regularly, academic researchers and CCT members have been able to engage in an ongoing trust-building process, resulting in a sustained research collaboration. The principles developed through this collaboration have influenced the research team's approach and have been carried forward to two additional projects.

While CCT members experienced positive outcomes from their study involvement, they also faced significant challenges to participation in the consultation team. Community members made financial, logistical, and emotional sacrifices to be involved. For many members, attending meetings meant arranging for childcare, traveling out of their way, sacrificing time with their families, and engaging in emotionally taxing conversations regarding their child's health needs and care experiences. The effort required of community members to engage with research consultation should not be minimized or taken for granted. By offering community partners fair compensation for their time and labor, researchers can demonstrate the degree to which they value their involvement. As one CCT member concisely stated, "We pay for what we value."

Research team members also experienced benefits and challenges in fostering meaningful engagement with the CCT. Benefits included the tangible improvements to the research protocol and materials detailed above, and a deeper understanding among research team members of the lived experiences of those who care for children with medical needs and how those experiences can inform research questions and study designs. Challenges included balancing institutional and funding agency regulations and rigors of a clinical trial design with the feedback received from the CCT. Researchers also had to be flexible in planning their schedules in order to be available to attend meetings at times convenient for community members, often outside of standard work hours. In addition, we encountered some challenges in recruiting a gender-balanced group to the CCT. Initially, the group was all women. We were able to recruit one male participant through a targeted effort through CCT members' personal connections.

In terms of NCGENES 2's overall recruitment goals, the study has exceeded its diversity recruitment goal, with over $70 \%$ of participants from underserved or underrepresented populations. Further, the ratio of participants appears to be retained throughout the clinical trial. While a direct correlation cannot be drawn between the engagement of the CCT and these outcomes, these results do align with the substantial body of literature evidencing the impact that early and consistent community engagement can have for recruitment for clinical trials and genomics research [25-27].

Prior research on community engagement in patient-centered outcomes research has found that stakeholder engagement is more likely to take place during the earlier stages of the research process, when study teams are defining research questions and planning the study approach. For example, prior research on genetic biobanks has focused on information gathering to learn about stakeholders' perspectives around biobanking research [28,29]. Similarly, a systematic review found that it was less common for stakeholder engagement to be sustained throughout data interpretation and dissemination phases [30]. This pattern was reiterated by some CCT members who reflected that prior experiences with researchers had tended to be one-time meetings, as if their involvement was simply 'to check a box'. The findings from the NCGENES 2 study emphasize the importance of an ongoing, reciprocal engagement process that enables stakeholders and academic researchers to form a meaningful partnership that encourages input as the study progresses and new challenges arise. This article builds on emerging evidence of the usefulness of community-academic partnerships in clinical genomics research [31] by offering practical guidance on the engagement process, specifically informed by the CCT's expertise as parents of children who have had genetic evaluation and/or who have medical needs. 
Future research should seek to establish mechanisms for long-term stakeholder involvement in the design and implementation of genomics research. The strategies outlined in this manuscript can help guide community engagement efforts, which can lead to more equitable representation of diverse patient populations in genomic sequencing research. Previous research has shown that meaningful community engagement requires flexible study protocols, which may disrupt traditional clinical research paradigms [32,33]. By being receptive to this form of disruption, making space for ongoing collaboration with community stakeholders, and making tangible changes to research protocols and materials based on stakeholder recommendations, genomics researchers have the opportunity to more fully incorporate patients across diverse racial, ethnic, and socioeconomic backgrounds.

\section{Future perspective}

Over the next 5 to 10 years, clinical applications for genomic information will continue to rapidly expand and impact a wide variety of patients and communities. Without the inclusion of diverse perspectives from members of various communities, these advances threaten to widen disparities in healthcare. As research strategies adapt to embrace the value of stakeholder-engaged approaches to medical genomics [34], our healthcare systems will need to develop the infrastructure to enable innovative changes to care delivery that are flexible and responsive to a wide array of patients and communities.

\section{Summary points}

- Historical underrepresentation and exploitation of people of color in medical research has undermined the accessibility and utility of health research studies for underserved populations.

- Research on best practices to improve diversity in genomics research is urgently needed to ensure that precision medicine is available and useful for all.

- Emerging research underscores the need to engage community members in substantive roles to inform research processes throughout the life cycle of a study.

- The NCGENES 2 study formed a Community Consult Team (CCT) made up of members from underserved and underrepresented groups who advised the research team throughout study development, on-going enrollment, and dissemination of findings and key messages.

- The research team used engagement strategies that built trust, demonstrated value, accommodated CCT members' schedules, and allowed for ongoing involvement in the study process.

- Recommendations by the CCT were imperative to designing recruitment and enrollment protocols, optimizing educational materials, and enhancing language accessibility.

- The community-academic collaboration was rewarding to CCT members due to their ongoing involvement in the NCGENES 2 study and the tangible impact of their contributions.

- Fair compensation of CCT members was essential to demonstrate the value of their perspectives for the research and to offset potential costs associated with their involvement.

- The NCGENES 2 team recommends researchers establishing mechanisms for long-term collaboration with community-based consultants to improve the design and implementation of genomics research and increase equitable representation of diverse patient populations.

Author contributions

I Griesemer, BS Staley, AF Lightfoot, L Bain, D Byrd, C Conway, TL Grant, B Leach, L Milko, N Porter, S Reid, G Smith, M Waltz, JS Berg, C Rini and JM O'Daniel made substantial contributions to the conception and design of the work. I Griesemer, BS Staley, AF Lightfoot, LM, M Waltz, C Rini and JM O'Daniel contributed to the analysis and interpretation of data for the work. All authors contributed to the writing and revising of the work for important intellectual content, give final approval of the version to be published, and agree to be accountable for all aspects of the work in ensuring that questions related to the accuracy or integrity of any part of the work are appropriately investigated and resolved.

Financial \& competing interests disclosure

Support for this research was provided by the National Human Genome Research Institute (NHGRI) [U01HG006487], the National Cancer Institute (NCI) [RFA-HG-12-009], the National Center for Advancing Translations Sciences [UL1TR002489] and the Agency for Healthcare Research and Quality (AHRQ) sponsored by The Cecil G. Sheps Center for Health Services Research, The University of North Carolina at Chapel Hill [T32 HS000032] (for I Griesemer). The authors have no other relevant affiliations or financial involvement with any organization or entity with a financial interest in or financial conflict with the subject matter or materials discussed in the manuscript apart from those disclosed.

No writing assistance was utilized in the production of this manuscript. 


\section{Ethical conduct of research}

All study procedures were approved by the University of North Carolina at Chapel Hill's Institutional Review Board (IRB), and participants provided informed consent to take part in NCGENES 2.

\section{Open access}

This work is licensed under the Attribution-NonCommercial-NoDerivatives 4.0 Unported License. To view a copy of this license, visit http://creativecommons.org/licenses/by-nc-nd/4.0/

\section{References}

Papers of special note have been highlighted as: $\bullet$ of interest

1. Stark Z, Dolman L, Manolio TA et al. Integrating genomics into healthcare: a global responsibility. Am. J. Hum. Genet. 104(1), 13-20 (2019).

2. Retterer K, Juusola J, Cho MT et al. Clinical application of whole-exome sequencing across clinical indications. Genet. Med. 18(7), 696-704 (2016).

3. Schwarze K, Buchanan J, Taylor JC, Wordsworth S. Are whole-exome and whole-genome sequencing approaches cost-effective? A systematic review of the literature. Genet. Med. 20(10), 1122-1130 (2018).

4. Delaney SK, Hultner ML, Jacob HJ et al. Toward clinical genomics in everyday medicine: perspectives and recommendations. Expert Rev. Mol. Diagn. 16(5), 521-532 (2016).

5. Lemke AA, Harris-Wai JN. Stakeholder engagement in policy development: challenges and opportunities for human genomics. Genet. Med. 17(12), 949-957 (2015).

- Provides a rationale for engaging stakeholders in genomics policy development and summarizes examples of stakeholder engagement efforts in the field.

6. Hindorff LA, Bonham VL, Brody LC et al. Prioritizing diversity in human genomics research. Nat. Rev. Genet. 19(3), 175 (2018).

- Summarizes the National Human Genome Research Institute's efforts to increase diversity in gemonics research studies and the genomic research work force.

7. Popejoy AB, Ritter DI, Crooks K et al. The clinical imperative for inclusivity: race, ethnicity, and ancestry (REA) in genomics. Hum. Mutat. 39(11), 1713-1720 (2018).

8. Hartzler A, Mccarty CA, Rasmussen LV et al. Stakeholder engagement: a key component of integrating genomic information into electronic health records. Genet. Med. 15(10), 792-801 (2013).

9. Amendola LM, Berg JS, Horowitz CR et al. The clinical sequencing evidence-generating research consortium: integrating genomic sequencing in diverse and medically underserved populations. Am. J. Hum. Genet. 103(3), 319-327 (2018).

10. Concannon TW, Grant S, Welch V et al. Practical guidance for involving stakeholders in health research. J. Gen. Intern. Med. 34(3), 458-463 (2019).

- Offers tools to guide researchers' stakeholder engagement processes and the evaluation of the impact of stakeholder engagement in health research.

11. Esmail L, Moore E, Rein A. Evaluating patient and stakeholder engagement in research: moving from theory to practice. J. Comp. Eff. Res. 4(2), 133-145 (2015).

- Provides guidance on how to measure and evaluate the value of stakeholder enegagement in research.

12. Gamble VN. A legacy of distrust: African Americans and medical research. Am. J. Prev. Med.9(6), 35-38 (1993).

13. Brandt AM. Racism and research: the case of the Tuskegee Syphilis Study. Hastings Cent. Rep. 8(6), 21-29 (1978).

14. Katz RV, Russell SL, Kegeles SS et al. The Tuskegee Legacy Project: willingness of minorities to participate in biomedical research. J. Health Care Poor Underserved. 17(4), 698 (2006).

15. Corbie-Smith G. The continuing legacy of the Tuskegee Syphilis Study: considerations for clinical investigation. Am. J. Med. Sci. 317(1), 5-8 (1999).

16. Wikler D. Can we learn from eugenics? J. Med. Ethics 25(2), 183-194 (1999).

17. Beskow LM. Lessons from HeLa cells: the ethics and policy of biospecimens. Annu. Rev. Genomics Hum. Genet. 17, 395-417 (2016).

18. Robinson JM, Trochim WM. An examination of community members', researchers' and health professionals' perceptions of barriers to minority participation in medical research: an application of concept mapping. Ethn. Health. 12(5), 521-539 (2007).

19. Samuel T. Standardizing a process to engage African Americans in health research: the Community Research Outreach Workers' Network (CROWN). Prog. Community Health Partnersh. 8(1), 109-116 (2014).

20. Durant RW, Davis RB, George DMMS, Williams IC, Blumenthal C, Corbie-Smith GM. Participation in research studies: factors associated with failing to meet minority recruitment goals. Ann. Epidemiol. 17(8), 634-642 (2007). 
21. Cotugna N, Vickery CE, Carpenter-Haefele KM. Evaluation of literacy level of patient education pages in health-related journals. J. Community Health 30(3), 213-219 (2005).

22. Foreman A, Lee K, Evans JP. The NCGENES project: exploring the new world of genome sequencing. N. C. Med. J. 74(6), 500-504 (2012).

23. Samuel CA, Lightfoot AF, Schaal J et al. Establishing New Community-Based Participatory Research Partnerships using the Community-Based Participatory Research Charrette Model: Lessons from the Cancer Health Accountability for Managing Pain and Symptoms Study. Prog. Community Health Partnersh. 12(1), 89-99 (2018).

24. Griesemer I, O'daniel J, Lightfoot A et al. Stakeholder engagement strategies to strengthen research on best practices for clinical genomic sequencing with underrepresented and underserved populations. Presented at: APHA's 2019 Annual Meeting and Expo, 2-6 November 2019.

25. Cohn EG, Husamudeen M, Larson EL, Williams JK. Increasing participation in genomic research and biobanking through community-based capacity building. J. Genet. Couns. 24(3), 491-502 (2015).

- Describes research outcomes from a community-based capacity building building process that can increase equity in genomic research participation.

26. Ewing A, Thompson N, Ricks-Santi L. Strategies for enrollment of African Americans into cancer genetic studies. J. Cancer Educ. 30(1), 108-115 (2015).

27. Spruill IJ. Enhancing recruitment of African-American families into genetic research: lessons learned from Project SuGar. J. Community Genet. 1(3), 125-132 (2010).

28. Staunton C, Tindana P, Hendricks M, Moodley K. Rules of engagement: perspectives on stakeholder engagement for genomic biobanking research in South Africa. BMC Med. Ethics. 19(1), (2018).

29. Beaton A, Hudson M, Milne M et al. Engaging Māori in biobanking and genomic research: a model for biobanks to guide culturally informed governance, operational, and community engagement activities. Genet. Med. 19(3), 345-351 (2017).

30. Concannon TW,, Fuster M, Saunders T et al. A systematic review of stakeholder engagement in comparative effectiveness and patient-centered outcomes research. J. Gen. Intern. Med. 29(12), 1692-1701 (2014).

31. Kaplan B,, Caddle-Steele C, Chisholm G et al. A culture of understanding: reflections and suggestions from a genomics research community board. Prog. Community Health Partnersh. 11(2), 161-165 (2017).

32. Dobransky-Fasiska D, Brown C, Pincus HA et al. Developing a community-academic partnership to improve recognition and treatment of depression in underserved African American and white elders. Am. J. Geriatr. Psychiatry 17(11), 953-964 (2009).

33. Ochs-Balcom HM, Jandorf L, Wang Y et al. "It takes a village": multilevel approaches to recruit African Americans and their families for genetic research. J. Community Genet. 6(1), 39-45 (2015).

- Describes a community-based participatory research process used to recruit African-American women and their relatives for a breast cancer genomics study.

34. Horowitz CR, Orlando LA, Slavotinek AM et al. The genomic medicine integrative research framework: a conceptual framework for conducting genomic medicine research. Am. J. Hum. Genet. 104(6), 1088-1096 (2019). 